\title{
ESTILO GERENCIAL Y SU INFLUENCIA EN LA COMUNICACIÓN DE EDUCACIÓN BÁSICA
}

\section{MANAGEMENT STYLE AND ITS INFLUENCE ON THE COMMUNICATION OF BASIC EDUCATION}

\author{
José Nicolás Isea Araque, Msc. \\ Máster en Gerencia y Liderazgo Educativo (Venezuela). \\ Centro de Formación y Divulgación Científica “Andrés Bello”, Venezuela. \\ nikoiseaa@gmail.com \\ Yusmany Mora de Isea, Msc. \\ Máster en Gerencia y Liderazgo Educativo (Venezuela). \\ Centro de Formación y Divulgación Científica “Andrés Bello”, Venezuela. \\ myjiret@gmail.com \\ Jesús Antonio Gómez Escorcha, PhD. \\ Doctor en Ciencias de la Educación (Venezuela). \\ Docente de la Universidad de Otavalo, Ecuador. \\ jgómez@uotavalo.edu.ec
}

\section{ARTÍCULO DE INVESTIGACIÓN}

Recibido: 15 de agosto de 2017

Aceptado: 30 de octubre de 2017

\section{RESUMEN}

El estudio tuvo como objetivo general analizar la influencia del estilo del gerente sobre la comunicación en la Escuela Bolivariana "Ciudad Varyná", municipio Barinas, estado Barinas, Venezuela. La misma se fundamentó en el paradigma cuantitativo, estudio de campo de nivel descriptivo. En cuanto a los instrumento de recolección de datos se usó la técnica de análisis de documental para conocer a profundidad las variables del estudio, la 
observación estructurada usada por el investigador para registrar los hechos de la problemática y para recabar la información se utilizó un cuestionario de escala tipo Likert dirigido a la muestra de estudio (20 docentes). Para la validez del instrumento se empleó la técnica de juicio de expertos y la confiabilidad a través del coeficiente Alfa de Crombach. Se obtuvo como resultado el uso inapropiado de la comunicación basado en un estilo gerencial autocrático por parte del directivo. Se concluyó que cada gerente de acuerdo a su estilo particular impacta de manera significativa en los procesos comunicacionales, en la educación básica la comunicación eficaz es una herramienta indispensable para transmitir lineamientos, promover objetivos favoreciendo el trabajo en equipo y de este modo garantizar la operatividad de las instituciones.

Palabras clave: gerencia, educación, comunicación

\section{ABSTRACT}

The study had as general objective to analyze the influence of the manager's style on the communication in the Bolivarian School "City Varyná", municipality Barinas, Barinas state, Venezuela. It was based on the quantitative paradigm, field study of descriptive level. As for data collection instruments, the documentary analysis technique was used to know in depth the variables of the study, the structured observation used by the researcher to record the facts of the problem and to collect the information a questionnaire was used Likert scale for the study sample (20 teachers). For the validity of the instrument was used the technique of expert judgment and reliability through the Alpha coefficient of Crombach. As a result, the inappropriate use of communication based on an autocratic managerial style was obtained by the manager. It was concluded that each manager, according to his / her particular style, has a significant impact on communication processes. In basic education, effective communication is an indispensable tool for transmitting guidelines, promoting objectives, fostering teamwork and thus ensuring the institutions.

Keywords: management, education, communication

ISSN: 1390-9320, Vol. 4, No.6, diciembre 2017 


\section{INTRODUCCIÓN}

La comunicación es un elemento indispensable que sostiene la sociedad, además, es un proceso que garantiza la transmisión de las ideas y facilita la armonía de las relaciones humanas. Por esta razón, dentro de la educación se requiere de gerentes que sean capaces de promover un adecuado proceso comunicacional que garantice la operatividad en las instituciones. En este sentido, se puede señalar "la comunicación es una herramienta que contribuye al consenso del personal, es decir, las organizaciones que mantienen sistemas de comunicación abiertos, da oportunidad al personal para que formen los procesos que están orientados a crear los valores de la organización" (Soto, 2016, p. 21). Partiendo de esta idea, se nutre así el sentido de pertenencia, las relaciones favorables y de este modo el logro de objetivos comunes.

Por otra parte, las instituciones escolares para su buen accionar en los diferentes departamentos dependen fundamentalmente de la actuación gerencial, que garantiza el adecuado uso de los recursos humanos y materiales, así como también del proceso formativo en general; sumado a los múltiples cambios que sufre el sistema educativo es donde surge la figura del gerente como principal protagonista. Por tal razón, se indica tocante a la gerencia educativa como "el proceso a través del cual se orienta y conduce la labor docente y administrativa de la escuela, y sus relaciones con el entorno para conseguir los objetivos institucionales mediante el trabajo de todos los miembros de la comunidad educativa" (Machado, 2017, p. 11). Es así, como el gerente educativo debe manejar el sistema que representa, la escuela que dirige o la red escolar que coordina, a fin de satisfacer las necesidades de los diferentes actores internos y externos vinculados a la institución.

En las escuelas del país se observan una serie de situaciones comunicacionales que afectan el logro de los objetivos institucionales, particularmente reina los diálogos, los consejos, las expresiones afectivas, pero también las humillaciones, las faltas de respeto continuo, agresión verbal, entre otras formas comunicativas que generan la creación de subgrupos parcelados de intereses particulares y que ponen en serio peligro las gestiones 
gerenciales socavando en muchos casos el ambiente de trabajo haciéndolo inestables, poco deseado y lleno de incertidumbre, en tal sentido se establece que "en las escuelas, la comunicación influye en el comportamiento y en el logro de las metas organizacionales" (Castro, 2016, p. 21).

En este orden de ideas, si no existe una comunicación por parte de la gerencia que estimule al docente, éstos se ven limitados en sus esfuerzos, lo cual puede generar conductas y comportamientos que afectan las actividades del maestro, tanto desde el punto de vista de la productividad, sus relaciones entre colegas y principalmente el no poder comprender las orientaciones brindadas desde la dirección del plantel para alcanzar objetivos comunes dentro de la comunidad educativa.

En el Estado Barinas también se genera a nivel gerencial serios problemas comunicacionales que afectan la acción operativa de los docentes y crea un ambiente laboral desfavorable para la función y el alcance de objetivos institucionales, así se expresa que "existen serias debilidades en el proceso de comunicación entre los docentes de las escuelas del Estado y esto influye muy seriamente en el clima laboral de las escuelas y en la capacidad productiva en el profesional de la docencia” (Rangel, 2013, p. 98). Además de la carencia por parte de los equipos gerenciales para crear estrategias que favorezcan la comunicación y permitan promover los planes, proyectos y objetivos de las instituciones.

Considerando los aspectos teóricos expuestos y partiendo de las entrevistas no estructuradas (observación directa), se puedo apreciar, que en la Escuela Bolivariana Ciudad Varyná, ubicada en la Parroquia Alto Barinas del Estado Barinas, se está generando una serie de situaciones conflictivas, ocasionada por los problemas comunicacionales en los cuales incurre la gerencia obviando las sugerencias por parte de los docentes, principalmente en la atención de exigencias y reclamos, siendo escasas las informaciones de las diferentes actividades de forma clara, oportuna creando desmotivación en el personal que labora en la escuela, trayendo como consecuencias una disminución considerable en la calidad del desempeño profesional y humano de los actores que fungen en ella, también desmotivación para la integración en las actividades propuestas, así como también un clima laboral no deseado, al no acatarse las líneas de acción que la gerencia señala. 
En tal sentido, la investigación se planteó el siguiente problema científico; ¿Cuál es la influencia que tiene el estilo del gerente sobre la comunicación en la Escuela Bolivariana Ciudad Varyná, del municipio Barinas, en el Estado Barinas durante el año 2011 ?

Atendiendo la situación que se presenta en esta institución, se plantea la siguiente investigación en cuanto al estilo gerencial y su influencia en la comunicación de educación la Escuela Bolivariana Ciudad Varyná, del municipio Barinas, del Estado Barinas; con lo que se debe responder una serie de interrogantes que permitan garantizar el desarrollo de la investigación las cuales son: ¿Cuál es la situación actual del gerente educativo en cuanto a las funciones que debe desempeñar en la Escuela Bolivariana Ciudad Varyná, municipio Barinas, Estado Barinas?, ¿Cómo es el estilo del gerente en la Escuela Bolivariana Ciudad Varyná? y ¿Cómo es la comunicación de la gerencia en la Escuela Bolivariana Ciudad Varyná? Para dar respuestas a las interrogantes se plantea el siguiente objetivo general:

Objetivo General: Analizar influencia que tiene el estilo del gerente sobre la comunicación en la Escuela Bolivariana Ciudad Varyná, del municipio Barinas, en el Estado Barinas durante el año 2011.

Objetivos Específicos: Describir el tipo de comunicación que emplea la gerencia en la Escuela Bolivariana Ciudad Varyná, municipio Barinas, Estado Barinas. Diagnosticar la situación actual del gerente educativo en cuanto a las funciones que debe desempeñar en la Escuela Bolivariana Ciudad Varyná, municipio Barinas, Estado Barinas, conocer el estilo gerencial que se desarrolla en la Escuela Bolivariana Ciudad Varyná

\section{REVISÓN TEÓRICA}

Por otra parte, en trabajo de investigación a nivel de Maestría en la Universidad Fermín Toro, titulado la comunicación efectiva entre el personal directivo y docente en la Escuela Básica Lourdes Rivas de Torres, orientado en un modelo cuasi cuantitativo, de campo, a un nivel de estudio de tipo documental y descriptivo, usando una muestra de 40 docentes, 
donde su objetivo general fue el determinar la efectividad de la comunicación entre el personal directivo y el personal docente de la Escuela Básica Lourdes Rivas de Torres, en el Municipio Barinas, quien pudo concluir que el proceso comunicativo no es efectivo entre el personal directivo y docente de la institución escolar, así como las relaciones interpersonales no son del todo integradoras, lo que hace desfavorable la integración y el trabajo en equipo afectando el logro de objetivos institucionales, el desempeño laboral y el clima laboral de la misma (Rivera, 2013).

Según esta evidencia, el mal uso de la comunicación por parte del personal directivo y docente de las instituciones escolares genera un impacto negativo en los colectivos que laboran en ella afectando el desempeño laboral dentro de las instituciones educativas y perturba el logro de los objetivos trazados por la misma.

\section{Gerencia}

Atendiendo este término relacionado con la gerencia se afirma que "es un cargo que ocupa el director de una empresa y tiene múltiples funciones, representar a la sociedad frente a terceros y coordinar los recursos a través de planeamiento, organización dirección y control a fin de lograr objetivos establecidos”. (Romero, 2005, p. 17). Ante este panorama, el complejo funcionamiento del gerente demanda de una serie de tareas que no puede dejar pasar por alto porque están incluidas en un ciclo bien orquestado.

\section{Funciones del gerente}

En cuanto a la función del gerente para conducir la institución se vale de funciones típicas como lo son: la planificación, organización, dirección y control de sus tareas. Al respecto, se plantea: "las funciones de los gerentes se deben enmarcar dentro del proceso administrativo, por lo tanto, las tareas gerenciales esenciales son planeación, organización, dirección y control" (Koontz, 2015, p.123). Las cuales se describen brevemente a continuación. 
Planificación: en esta fase el gerente con su equipo decide qué y cómo hacerlo para convertir la institución en un centro de excelencia pedagógica.

Organización: implica el diseño de la estructura formal para el desarrollo de la gestión de la escuela, facilitando la integración y coordinación de las actividades docentes, alumnos y otros agentes; así como, el empleo de los recursos para desarrollar los procesos, programas y proyectos que involucran tanto el trabajo como las funciones, a través de una jerarquía de autoridad y responsabilidad entre los actores y el entorno.

Dirección: asociada con el liderazgo, la motivación y la creación de un clima organizacional por parte del directivo, que integre las potencialidades de los diferentes sujetos, a partir del compromiso de todos con el proyecto educativo para mejorar la docencia y la administración de los recursos de la escuela. La dirección garantiza la motivación, la guía, y orientar las decisiones para el logro de los objetivos de la organización.

Control: consiste en asegurar la ejecución de la programación de acuerdo al esquema de responsabilidad y distribución del trabajo que se diseñó para el logro de los objetivos y las metas y actividades, con el fin de evaluar continuamente los resultados.

\section{Estilos gerenciales}

Existen estilos gerenciales vinculados con el carácter de cada liderazgo según entre ellos se encuentra el autócrata, es donde "el gerente centraliza las decisiones e impone órdenes al grupo, determina los pasos para seguir a medida que sean necesarios e imprevisibles para el grupo, fija la tarea de cada uno y asigna el compañero de trabajo, es dominante" (Cerna, 2015, p. 22). De acuerdo al autor, este gerente es quien asume toda la responsabilidad de la toma de decisiones, inicia las acciones, dirige, motiva y controla a todo el personal, parte del principio que todos le obedezcan. El estilo autocrático no es el más recomendado para las gerencias educativas, ya que tienden a anular a las demás personas, restándole importancia y negándole la oportunidad de crecer, mostrar y desarrollar sus potencialidades.

ISSN: 1390-9320, Vol. 4, No.6, diciembre 2017 
Por su parte el estilo participativo: utiliza la consulta para ejercer su liderazgo. No delega su derecho a tomar decisiones finales señalando directrices específicas a sus subalternos, pero consulta ideas y opiniones. En este orden de ideas, "este estilo consulta a sus subordinados las acciones y decisiones probables y alienta su participación, considera tanto a aquellos que no participan como a los que si lo hacen; toman decisiones por sí solo pero consulta a todos" (Fernández, 2015, p.83). De igual forma, este estilo gerencial muestra una fuerte preocupación por el personal que tiene a su cargo, alienta a todos a tomar parte activa en las operaciones de la institución. Este gerente tiene un feliz equilibrio entre controlar y no estar ocupado.

El estilo situacional se define como aquel que "pueden analizar una situación determinada para luego adoptar un estilo apropiado a esa situación, así poder cumplir con la tarea exitosamente" (Colino, 2015, p.17). El análisis de esta situación va a depender principalmente de la madurez de los empleados para comprender y abocar esfuerzos por alcanzar los objetivos de la institución. Es importante señalar, que este estilo gerencial se basa en mantener un equilibrio entre dos comportamientos que pueda ejercer el director, en primer lugar, la exigencia de la tarea que pueda surgir y el nivel de desarrollo (madurez) de los empleados.

En relación a gerente transformacional, se indica que "son los que articulan una visión e inspiran a sus seguidores. Poseen la capacidad de motivar, moldear la cultura organizacional y de crear un ambiente favorable para el cambio institucional" (Fernández, 2015, p. 38). Es importante señalar que este estilo gerencial no se caracteriza por la manera en que toma las decisiones, sino en la medida en que las personas que conforman el colectivo de empleados lo reconozcan por su filosofía, capacidades; además de buscar potenciar el desarrollo de sus colaboradores, sus capacidades, motivaciones y valores, más no solo en el desempeño. Establece metas y objetivos en su intento de hacer de sus seguidores líderes potenciales que lo puedan suceder.

Sobre la base de estas teorías, el gerente educativo que requiere las instituciones escolares debe ser aquel que transmite confianza, posee una visión clara de lo que se quiere lograr, 
aplica estrategias para producir y aceptar los cambios dentro del personal, crea entusiasmo, coopera y motiva al colectivo en general.

\section{Gerencia Educativa}

En este orden de ideas, tocante a la gerencia educativa como "El proceso de planificación, organización, ejecución, control y supervisión de todos los recursos materiales, humanos y de las actividades de aprendizaje para alcanzar con éxito los objetivos y las metas en un diseño curricular" (Camacho, 2013, p.26). Entendiendo que la meta principal de toda institución educativa y, de hecho, de su administración es la de conseguir un beneficio social como lo es la formación de los recursos humanos de la más alta excelencia y calidad profesional. Una gerencia educativa que desee iniciar un cambio debe, primeramente, modernizar la concepción y práctica de los enfoques, tendencias y procesos con base en la construcción social, la concertación, la flexibilidad, la eficacia y la eficiencia en los procesos, a fin de promover la autonomía, la participación y la formación para la toma de decisiones dentro de los miembros y las distintas dimensiones que integran la institución (centro, aula, comunidad) el centro es el lugar del encuentro, de coordinación entre los actores; el aula, es el lugar donde se establece las relaciones de enseñanza y aprendizaje entre los estudiantes y los educadores; la comunidad es el entorno que rodea en centro educativo de donde provienen los alumnos y sus familias.

\section{Comunicación en las organizaciones}

Las formas de comunicación existenciales son tan diversas que entrañan una infinidad de fenómenos. El término comunicación hace referencia al acto, al proceso y al resultado de la transferencia de gestos, informaciones, objetos. Por eso se puede decir que "la comunicación es un hecho fundante de las relaciones en las organizaciones, las cuales se entablan, mantienen y fomentan a través de ella". (Muñoz, 2016, p. 20). El autor considera que el acto comunicativo cuando se desarrolla entre sujetos sociales más complejos (públicos, instituciones, gobiernos, reparticiones), es más factible que las complicaciones propias de este acto se agudizan aún más.

ISSN: 1390-9320, Vol. 4, No.6, diciembre 2017 


\section{Comunicación asertiva}

Ser asertivo significa el valor que tiene una persona de saber usar la percepción y la comunicación. En tal sentido, lo que se quiere destacar y dejar claro es que, en un mensaje, lo importante no es el "qué", sino el "cómo" se expresan las ideas, sentimientos, opiniones, una opción asertiva es madurez y efectividad personal.

Por eso, la comunicación asertiva: "es una opción distinta a la respuesta pasiva y opuesta a la agresividad que evita conflictos y posterga las necesidades del medio y crea innecesarias tensiones. "Con la asertividad el individuo puede expresar, dirigir y cambiar sus pensamientos y conductas". (Camacho, 2013, p.172). Así también, en este orden de ideas, indica que la comunicación asertiva es "La capacidad que se tiene de enfrentar de manera efectiva los conflictos que se plantean en el trabajo y en la capacidad de poder expresarse sin lesionar no los derechos y sentimientos propios, ni los de los individuos influenciados por el líder" (Ruiz, 2014, p.73).

Es evidente que la comunicación puede crear un clima de tensión o no, se debe procurar utilizar una comunicación asertiva donde se pueda dar a conocer las ideas de manera clara, cargada de las emociones que cada individuo pueda poseer y de este modo influir en la conducta de los demás, es decir, la comunicación es la herramienta que todo gerente debe saber utilizar para la transmisión adecuada de sus líneas de acción dentro de la organización.

\section{Características de la Comunicación Efectiva}

Para Camacho (2013). La comunicación efectiva tiene las siguientes características:

Es clara: la comunicación debe presentarse de una forma en que todos los receptores entiendan el mensaje. Esto significa que el lenguaje y el contenido, deben ser sencillos, para ello debe usar expresiones comunes, ser objetivo y lo más concreto posible.

ISSN: 1390-9320, Vol. 4, No.6, diciembre 2017 
Está bien fundamentada: antes de establecer una comunicación es indispensable tener una idea clara del mensaje, para ello es necesario estar bien informado de lo que se quiere transmitir, ya que si el emisor no está claro en su mensaje va a ocasionar confusión en los receptores de la información.

Unifica los Criterios: el mensaje transmitido no debe ser contradictorio en sus partes, ni en su contenido, esto significa que debe llevar en sí una decisión unánime de lo que se transmite y la misma no debe estar en contradicción con los fines que persigue la organización.

Es cortés: como miembro de la organización, cada persona cumple funciones específicas dentro de la misma; es por ello que todos los empleados, sin excepción, deben ser tomados con buena educación, amabilidad y con un trato agradable.

Está bien distribuida y es oportuna: es necesario asegurarse que la comunicación llegue a todos los receptores a la que va dirigida para evitar que una persona no se sienta partícipe en algún asunto de importancia. Asimismo debe procurarse que el mensaje llegue en el momento oportuno, evitando la comunicación extemporánea, bien por adelantado o por retraso.

Puede evaluarse: es de vital interés que el agente transmisor del mensaje siga el curso de la comunicación y conozca la reacción producida en el receptor, esto debe tenerse presente con el propósito de hacerla efectiva y que dé resultados positivos. En toda organización, quien tenga la responsabilidad de dirigir al personal debe tener presente que los aspectos antes señalados son indispensables en el proceso de transmitir información al resto de los empleados; de esta manera, se podrá lograr una comunicación efectiva con los trabajadores y, como consecuencia de ésta, una mejor coordinación de las operaciones de la misma. La coordinación se logra a través de la comunicación, la cual mantiene a todo el personal unido en el logro de los propósitos comunes; en este sentido, es fundamental la formación de equipos de trabajo para que los mismos sean cooperativos. 


\section{MATERIALES Y MÉTODOS}

La investigación está sustentada metodológicamente en el modelo cuantitativo, al respecto esta, "usa recolección de datos para probar hipótesis con base en la medición numérica y el análisis estadístico descriptivo, con el uso de tabla de distribución de frecuencia y gráficos de sectores”. (Hernández, Fernández y Baptista, 2014, p.76). Por otro lado, el método de investigación es deductivo, es señalado "cuando se aspira demostrar a través de la lógica pura la conclusión en su totalidad a partir de una premisa de tal manera que garantice la totalidad de la misma". (Molina, 2010, p.56).

Es decir, cuando se aspira demostrar, mediante la lógica para la ejecución de su totalidad a partir de las premisas, de manera que se garantice la veracidad de la conclusión. Es de carácter descriptiva, por cuanto la misma permite observar y medir el fenómeno estudiado, por lo que señala que "los estudios descriptivos buscan especificar las propiedades importantes de personas, grupos, comunidades o cualquier otro fenómeno que sea sometido a análisis" (Cabino, 2010, p.36).

De igual manera, es una investigación de campo porque se realizó en el propio sitio donde se encuentra el objeto de estudio; fundamentándose de la siguiente manera, "la investigación de campo surge como una estrategia que cumple el investigador basado en métodos que permite recoger los datos en forma directa de la realidad donde se presenta". (Tamayo y Tamayo, 2010, p.32). Para la recolección de la información pertinente al problema, se utilizó la técnica de análisis de documental para conocer a profundidad las variables del estudio, observación estructurada usada por el investigador para conocer los hechos de la problemática. Para recabar la información se utilizó un cuestionario de escala tipo Likert dirigido a la muestra de estudio (20 docentes).

\section{ANÁLISIS DE RESULTADOS Y DISCUSIÓN}

A continuación, se presenta el análisis e interpretación de los resultados, derivado de la aplicación del instrumento a los docentes de la Escuela Bolivariana Ciudad Varyná del Municipio Barinas, del Estado Barinas.

ISSN: 1390-9320, Vol. 4, No.6, diciembre 2017 
Tabla 1. Distribución de la opinión de los docentes con relación a la Variable: Gerencia. Dimensión: Funciones del Gerente.

\begin{tabular}{|c|c|c|c|c|c|c|c|}
\hline \multirow[t]{2}{*}{$\mathbf{N}^{\circ}$} & \multirow[t]{2}{*}{ İtems } & \multicolumn{2}{|c|}{ Siempre } & \multicolumn{2}{|c|}{ Alg. Veces } & \multicolumn{2}{|c|}{ Nunca } \\
\hline & & $\mathbf{F a}$ & $\%$ & $\mathbf{F a}$ & $\%$ & $\mathbf{F a}$ & $\%$ \\
\hline 1 & El directivo planifica las actividades a realizarse| & 16 & $80 \%$ & 4 & $20 \%$ & 0 & $0 \%$ \\
\hline 2 & $\begin{array}{l}\text { El directivo organiza grupos de trabajos para que se } \\
\text { cumplan las actividades planificadas }\end{array}$ & 13 & $65 \%$ & 7 & $35 \%$ & 0 & $0 \%$ \\
\hline 3 & $\begin{array}{l}\text { El directivo tiene la capacidad de dirigir las actividades } \\
\text { propias de la institución }\end{array}$ & 15 & $75 \%$ & 5 & $25 \%$ & 0 & $0 \%$ \\
\hline 4 & $\begin{array}{l}\text { La dirección del plantel verifica que se cumpla con las } \\
\text { actividades planificadas durante año escolar }\end{array}$ & 9 & $45 \%$ & 6 & $30 \%$ & 5 & $25 \%$ \\
\hline
\end{tabular}

Fuente: Isea, 2011.

Gráfico 1. Distribución Porcentual del instrumento aplicado a los docentes. Variable: Gerencia.

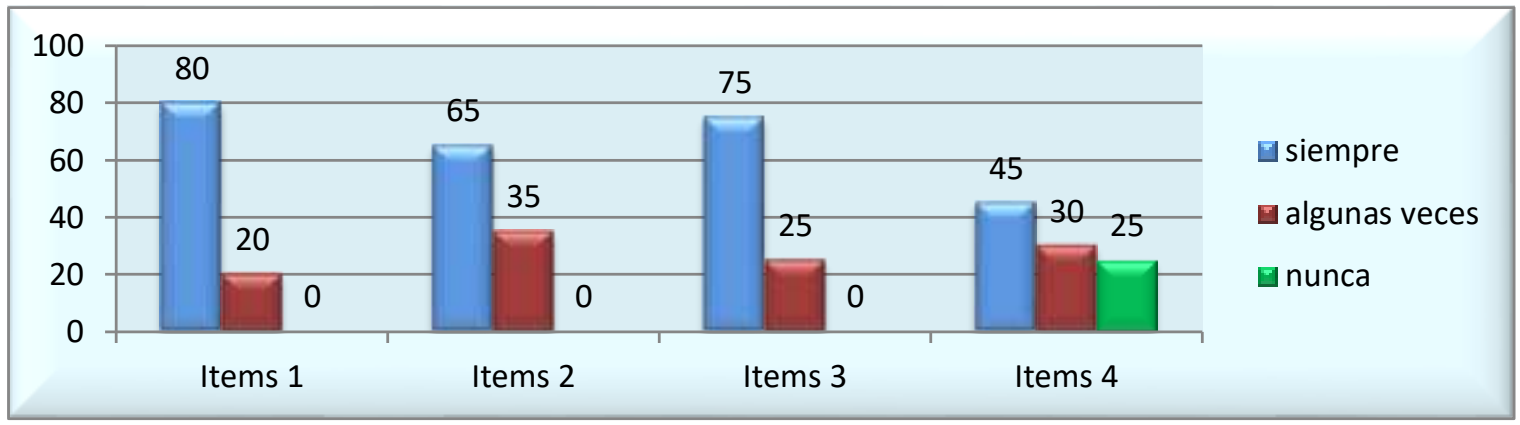

Fuente. Isea, 2011.

En relación al cuadro 1, gráfico 1, en el ítem 1, se pudo evidenciar que el ochenta por ciento $(80 \%)$ de los docentes entrevistados respondió "Siempre" el directivo planifica las actividades a realizarse, y el veinte por ciento (20\%) manifestó "Algunas Veces". En el ítems 2 , el sesenta y cinco por ciento (65\%) señaló "Siempre" el directivo organiza grupos de trabajos para que se cumplan las actividades planificadas mientras el treinta y cinco por ciento (35\%) restante opinó "Algunas veces". En el ítems 3, el setenta y cinco $(75 \%)$ acotó que "Siempre" el directivo tiene la capacidad de dirigir las actividades propias de la institución, mientras el otro veinte cinco por ciento (25\%) "Algunas Veces". Por otro lado, el cuarenta y cinco por ciento (45\%) manifestó que "Siempre" la dirección del plantel verifica 
que se cumpla con las actividades planificadas durante año escolar, el treinta por ciento $(30 \%)$ indica "Algunas Veces y el veinte cinco por ciento $(25 \%)$ que nunca.

Se evidencia en esta dimensión, que existe una tendencia porcentual bien marcada en su mayoría a la alternativa "Siempre", lo que permite inferir que el gerente de la institución cumple con la planificación de actividades conjuntas con el personal docente. De igual manera el directivo sí está dirigiendo adecuadamente las actividades propias de la institución, como resultado existen grupos organizados que verifiquen el cumplimiento de las actividades pautadas.

Al respecto se puede decir que, "la función de los gerentes debe estar enmarcadas dentro del proceso administrativo, por lo tanto, la principal tarea de un gerente debe ser: planificar, organizar, dirigir y controlar” (Koontz, 2015, p.123). De acuerdo a lo citado por el autor, es importante destacar que se deben establecer indicadores, criterios y un sistema de información para evaluar y retroalimentar al gerente sobre el avance y nivel de cumplimiento de objetivos, metas y actividades; todo ello con el fin de valorar continuamente los resultados e impactos en términos de nivel de rendimiento y de los procesos docentes y administrativos.

Tabla 2. Distribución de la opinión de los docentes con relación a la Variable: Estilo gerencial. Dimensión: Estilos gerenciales.

\begin{tabular}{|c|c|c|c|c|c|c|c|}
\hline \multirow[t]{2}{*}{$\mathbf{N}^{\circ}$} & \multirow[t]{2}{*}{ Items } & \multicolumn{2}{|c|}{ Siempre } & \multicolumn{2}{|c|}{ Alg. Veces } & \multicolumn{2}{|c|}{ Nunca } \\
\hline & & $\mathbf{F a}$ & $\%$ & $\mathbf{F a}$ & $\%$ & $\mathbf{F a}$ & $\%$ \\
\hline 5 & $\begin{array}{l}\text { ¿El directivo toma decisiones relacionadas con las } \\
\text { funciones del personal sin consultar con sus } \\
\text { compañeros de trabajo? }\end{array}$ & 17 & $85 \%$ & 3 & $15 \%$ & 0 & $0 \%$ \\
\hline 6 & $\begin{array}{l}\text { ¿El directivo se vincula activamente en las diferentes } \\
\text { actividades planificadas dirigiéndolas para garantizar su } \\
\text { cumplimiento? }\end{array}$ & 15 & $75 \%$ & 5 & $25 \%$ & 0 & $0 \%$ \\
\hline 7 & $\begin{array}{l}\text { ¿El gerente delega responsabilidades y tareas a sus } \\
\text { compañeros para la realización de actividades } \\
\text { especiales? }\end{array}$ & 3 & $15 \%$ & 5 & $25 \%$ & 12 & $60 \%$ \\
\hline
\end{tabular}

Fuente: Isea, 2011

ISSN: 1390-9320, Vol. 4, No.6, diciembre 2017 
Gráfico 2. Distribución porcentual del instrumento aplicado a los docentes. Variable: Gerencia

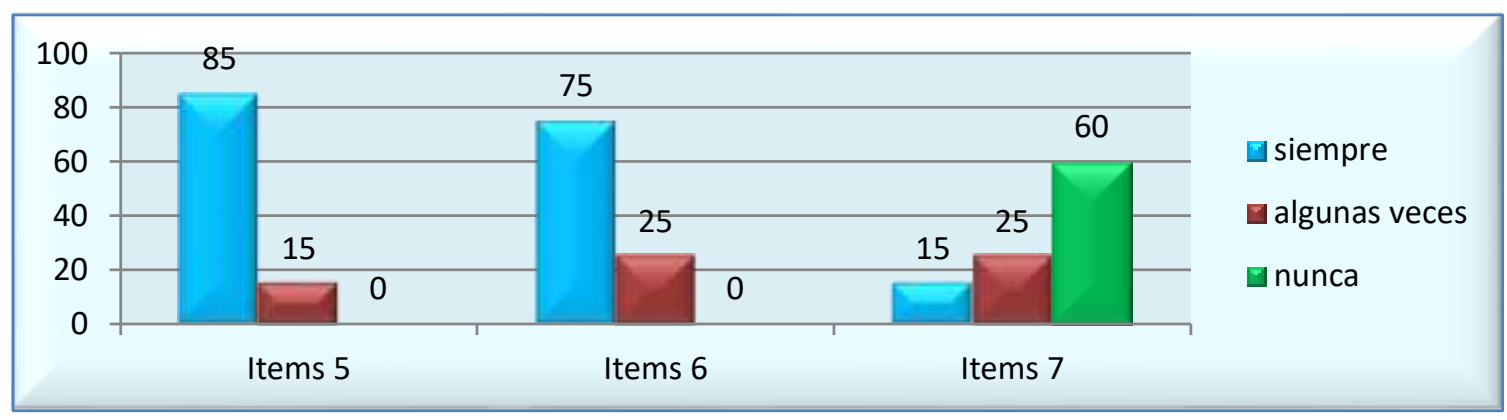

Fuente: Isea, 2011

En relación al cuadro 2, gráfico 2, en el ítem 5, se pudo observar que el ochenta y cinco por ciento (85\%) de los docentes señalaron "Siempre" el directivo toma decisiones relacionadas con las funciones del personal sin consultar con sus compañeros de trabajo, mientras el quince por ciento (15\%) "Algunas Veces". En el ítem 6, setenta y cinco por ciento $(75 \%)$ manifiestan "Siempre" el directivo se vincula activamente en las diferentes actividades planificadas dirigiéndolas para garantizar su cumplimiento y el veinte cinco por ciento (25\%) "Algunas Veces". Por otro lado, en el ítem 7, quince por ciento (15\%) "Siempre" el gerente delega responsabilidades y tareas a sus compañeros para la realización de actividades especiales, el veinte cinco por ciento (25\%) indicaron que "A veces" y el sesenta por ciento $(60 \%)$ que "Nunca".

En esta dimensión se pudo observar que existe una tendencia muy elevada en la alternativa "Siempre" en las dos primeras opciones, contrariamente con el resultado de la última pregunta donde se refleja mayor porcentaje en la alternativa "Nunca". En tal sentido, se evidencia que el personal de esta escuela percibe que la gerencia toma decisiones en relación al personal sin realizar ninguna consulta con ellos, al mismo tiempo consideran que el directivo se vincula en las diferentes actividades con el propósito de controlarlas y de ese modo poder garantizar su cumplimiento, además, los docentes indican que la gerencia no delega responsabilidades y tareas a sus compañeros para la realización de actividades 
especiales, considerando el estilo del gerente de esta escuela como un directivo con características autocráticas.

En tal sentido, "el gerente autocrático ordena y espera obediencia, es dogmático y positivo y dirige mediante la capacidad de retener o conceder recompensas o castigos". (Ariza, 2015, p. 14). Partiendo de las ideas del autor, no es conveniente que los gerentes institucionales desarrollen este estilo dentro de las escuelas ya que limita el accionar activo de los demás profesionales de la docencia.

Tabla 3. Distribución de la opinión de los docentes con relación a la Variable: Comunicación. Dimensión: Asertividad.

\begin{tabular}{|c|c|c|c|c|c|c|c|}
\hline \multirow[t]{2}{*}{$\mathbf{N}^{\circ}$} & \multirow[t]{2}{*}{ Ítems } & \multicolumn{2}{|c|}{ Siempre } & \multicolumn{2}{|c|}{ Alg. Veces } & \multicolumn{2}{|c|}{ Nunca } \\
\hline & & $\mathbf{F a}$ & $\%$ & $\mathrm{Fa}$ & $\%$ & $\mathrm{Fa}$ & $\%$ \\
\hline 8 & $\begin{array}{l}\text { ¿La comunicación que practica la gerencia es clara y } \\
\text { favorece el logro de los objetivos institucionales? }\end{array}$ & 1 & $5 \%$ & 17 & $85 \%$ & 2 & $10 \%$ \\
\hline 9 & $\begin{array}{l}\text { ¿Existe una comunicación efectiva entre el personal } \\
\text { docente y la gerencia de la escuela? }\end{array}$ & 0 & $0 \%$ & 14 & $70 \%$ & 6 & $30 \%$ \\
\hline 10 & $\begin{array}{l}\text { ¿La gerencia se comunica haciendo uso de las normas } \\
\text { de cortesía? }\end{array}$ & 5 & $25 \%$ & 9 & $45 \%$ & 6 & $30 \%$ \\
\hline
\end{tabular}

Fuente: Isea, 2011

Grafico 3. Distribución porcentual del instrumento aplicado a los docentes. Variable: Comunicación.

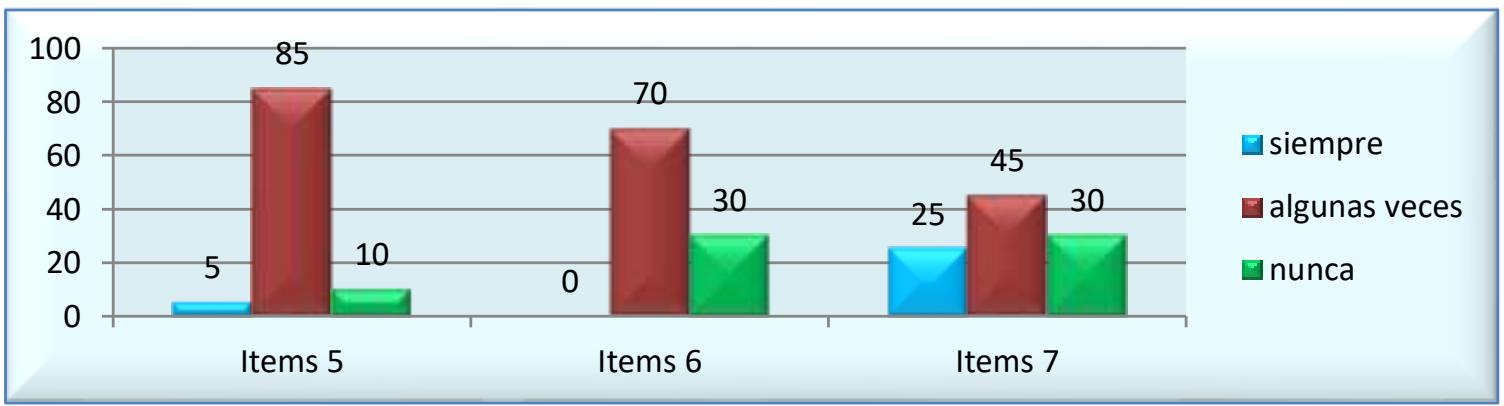

Fuente: Isea, 2011

En relación al cuadro 3, gráfico 3, en el ítem 8, se pudo evidenciar que el cinco por ciento $(5 \%)$ de los docentes entrevistados manifestó que "Siempre" la comunicación que practica la gerencia es clara y favorece el logro de los objetivos institucionales; por otra parte, ochenta y cinco por ciento (85\%) señaló "Algunas Veces" y el diez por ciento (10\%) 
manifestó “Nunca”. En el ítems 9, el setenta por ciento (70\%) señaló “Algunas veces" existe una comunicación efectiva entre el personal docente y la gerencia de la escuela y treinta por ciento (30\%) restante opinó "Nunca". En el ítems 10, el veinticinco por ciento (25\%) acotó que "Siempre" la gerencia se comunica haciendo uso de las normas de cortesía, mientras el otro cuarenta y cinco por ciento (45\%) "Algunas Veces" y el treinta por ciento $(30 \%)$ restante dijo "Nunca".

Partiendo de los datos obtenidos, se evidenció en esta dimensión, que existe una tendencia porcentual bien marcada en su mayoría a la alternativa "Algunas veces", lo que permite inferir que el gerente de esta institución pocas veces usa una buena comunicación lo que dificulta el logro de los objetivos institucionales; de igual manera, se constató que la comunicación no es efectiva entre el personal docente y la gerencia, lo que ocasiona como consecuencia el incumplimiento de las tareas tales como; la planificación, organización, dirección y control.

En contraste a esta situación, el gerente en términos conceptuales representa lo siguiente; "es un cargo que ocupa el director de una empresa y tiene múltiples funciones, representar a la sociedad frente a terceros y coordinar los recursos a través de planeamiento, organización dirección y control a fin de lograr objetivos establecidos". (Romero, 2005, p.17), de lo antes citado, la figura del gerente o director de un plantel, demanda un conjunto de tareas que contribuyen con el liderazgo, la motivación, un clima organizacional adecuado que favorece al docente para cumplir con las responsabilidades de la institución de manera mancomunada y eficiente.

Aunado a este planteamiento, se hace referencia a la comunicación asertiva como aquella que "Está dirigida a obtener resultados eficaces y adecuarse a las circunstancias", (Storey, 2016, p.45), en otras palabras, son claras las expresiones de los objetivos y firmes en la intensión de lograrlos, sin ser agresivo ni descartes, pues toma en cuenta los deseos y derechos de los demás sin imponer su voluntad a la fuerza. A través del uso de la comunicación efectiva la gerencia pudiera enfocarse en aplicar un conjunto de actividades que permita entrenar a los individuos en la cual se defienden sus legítimos derechos sin agredir ni ser agredidos.

ISSN: 1390-9320, Vol. 4, No.6, diciembre 2017 


\section{CONCLUSIONES}

En cuanto a las funciones del gerente se concluye en que los directivos de esta institución desarrollan adecuadamente los procesos gerenciales, principalmente porque se involucran en la planificación de actividades generalizadas y por lo general organizan grupos de trabajos para que se cumplan dichas actividades, por otra parte la gerencia tiene de la capacidad de dirigir las actividades propias de la institución, y muestra buenos niveles de control sobre el personal evidenciándose esto en que el directivo verifica que se cumpla con las actividades planificadas durante año escolar, esto permite decir que los procesos gerenciales llevados por el director son apropiados.

Asimismo, el estilo de gerencia que emplea el director en la Escuela Bolivariana Ciudad Varyná, es de carácter autocrático, pues su perfil se vincula con las teorías que lo describen de ese modo, este gerente no socializa las funciones de sus docentes a los cuales no les consulta para el momento de tomar decisiones, por otra parte el gerente se vincula activamente en las diferentes actividades planificadas dirigiéndolas para garantizar su cumplimiento, por lo general no delega esta función, lo que es evidente que requiere tener el control generalizado de las diferentes actividades que se planean en la escuela.

También, la comunicación que practica la gerencia en la Escuela Bolivariana Ciudad Varyná no es clara lo que no favorece el logro de los objetivos institucionales; creándose de este modo algunas interrupciones, falta de atenciones por parte de los docentes a las indicaciones del directivo, lo que permite señalar que en esta institución no existe una comunicación efectiva entre el personal docente y la gerencia, colocando en gran riesgo el logro de los objetivos institucionales, pudiéndose generar serios conflictos de carácter personal por encima de lo laboral por errores comunicacionales en los que está incurriendo la gerencia.

Por otra parte, el estilo personal de cada gerente impacta de manera significativa en los procesos comunicacionales, en este caso la manera autocrática de dirigir los procesos administrativos y pedagógicos en esta institución ha marcado la manera de comunicarse 
entre los diferentes miembros de la comunidad educativa. No es conveniente que los gerentes institucionales desarrollen este estilo dentro de las escuelas ya que limita el accionar activo de los demás profesionales de la docencia.

\section{REFERENCIAS BIBLIOGRÁFICAS}

Ariza, V. (2015). Líderes transformacionales en la compañía telefónica en sus cargos gerenciales (Bachelor's thesis, Universidad Militar Nueva Granada).

Camacho, M. (2013). El Enfoque Humanista en la Gerencia. Venezuela editorial fondo UNELLEZ.

Castro, E. (2016). Conflicto y comunicación informal en las organizaciones educativas. Omnia.

Cerna Rodríguez, K. (2015). El estilo de liderazgo de los gerentes y su influencia en el desempeño laboral del personal en una empresa de almacenes generales de la ciudad de Cajamarca.

Colino Toston, M. J. (2015). Conductas de liderazgo y Satisfacción Laboral.

Fernández, I (2015). Un análisis del fenómeno del liderazgo en la empresa= An analysis of the phenomenon of business leadership.

Hernández, Fernández y Baptista (2014). Metodología de la investigación. México: Editorial Mc Graw Hill.

Koontz, H. (2015). Habilidades del gerente educativo. Madrid, España: Editorial Gymmos.

Machado, M. (2017). Modelo de Gestión Gerencial del Sector Educativo en un Contexto Descentralizado. SINOPSIS EDUCATIVA. Revista venezolana de investigación.

Molina F, (2010). El proceso de investigación. Editorial Pardo. Barcelona-España.

Muñoz Y. (2016). Estrategias de comunicación organizacional y su relación con los estilos de liderazgo (Bachelor's thesis, Universidad Militar Nueva Granada).

Rangel, N. (2013). La comunicación y su Referencia en el Clima Organizacional_de la Escuela Bolivariana San Isidro. Barinas, Venezuela.

Rivera, S. (2013). Efectividad de la Comunicación Entre el Personal Directivo y el Personal Docente de la Escuela Básica Lourdes Rivas de Torres, en el Municipio Barinas. Trabajo de grado de Maestría no publicada Universidad Fermín Toro Lara. 
Romero, G. E. (2005). Papel del gerente líder venezolano: Gerencia vs. Liderazgo.

Ruiz, N. (2014). Satisfacción laboral y las dimensiones de asertividad en operarios de una organización privada de hidrocarburos. Talara, Piura, 2012.

Sabino C. (2010). El proceso de la Investigación. Editorial Panapo. Caracas-Venezuela.

Soto, C. (2016). Liderazgo en relación a la comunicación interna de las Empresas Familiares. Caso: Model Plac SRL (Doctoral dissertation).

Storey, R. (2016). El arte de la comunicación eficaz y persuasiva. Parkstone International.

Tamayo y Tamayo (2010). El proceso de la investigación científica. México D.F: Editorial LIMUSA. 